\title{
The Neural Representation of Self is Recapitulated in the Brains of Friends: A Round-Robin fMRI Study
}

\author{
Robert S. Chavez ${ }^{1}$ and Dylan D. Wagner ${ }^{2}$ \\ ${ }^{1}$ Department of Psychology, University of Oregon, Eugene, Oregon \\ ${ }^{2}$ Department of Psychology, The Ohio State University, Columbus, Ohio
}

\begin{abstract}
Humans continually form and update impressions of each other's identities based on the disclosure of thoughts, feelings, and beliefs. At the same time, individuals also have specific beliefs and knowledge about their own self-concept. Over a decade of social neuroscience research has shown that retrieving information about the self and about other persons recruits similar areas of the medial prefrontal cortex (MPFC), however it remains unclear if an individual's neural representation of self is reflected in the brains of well-known others or if instead the two representations share no common relationship. Here we examined this question in a tight-knit network of friends as they engaged in a round-robin trait evaluation task in which each participant was both perceiver and target for every other participant and in addition also evaluated their self. Using functional magnetic resonance imaging and a multilevel modeling approach, we show that multivoxel brain activity patterns in the MPFC during a person's self-referential thought are correlated with those of friends when thinking of that same person. Moreover, the similarity of neural self/other patterns was itself positively associated with the similarity of self/other trait judgments ratings as measured behaviorally in a separate session. These findings suggest that accuracy in person perception may be predicated on the degree to which the brain activity pattern associated with an individual thinking about their own selfconcept is similarly reflected in the brains of others.
\end{abstract}

self-knowledge | fMRI | personality judgement | traits | social networks

DOI: $10.1037 / p s p a 0000178$

Correspondence: rchavez@uoregon.edu

NOTE: The current manuscript is a preprint and may not perfectly reflect the final article.

Perhaps the most unique of human abilities is the capacity to introspect about our own thoughts and motives as well as those of other persons. Classic work in social and personality psychology has demonstrated that there is a strong relationship between a target's self-reported ratings of their own traits and that of their peers. For example, researchers have found that self/peer trait-judgment ratings corresponded highly with one another for observable traits (John \& Robins, 1993), especially when the target and peer are more similar to each other (Funder \& Colvin, 1988). For less observable traits (e.g., emotional stability) accuracy is more dependent on intimacy and level of friendship (Connelly \& Ones, 2010). Moreover, aggregate ratings across a set of peers, each of whom may know the target in a different way, have a higher correlation to the individual's own ratings of themselves compared to when using anyone of their peers ratings individually (McCrae \& Costa, 1987) and are also a better predictor of a target's behavior than individual peer ratings (Kolar, Funder, \& Colvin, 1996).

In recent years, functional neuroimaging studies have shown that self-referential thought and person perception engage common areas of the medial prefrontal cortex (MPFC) across a range of different tasks (Gobbini, Gors, Halchenko, Hughes, \& Cipolli, 2013; Hughes \& Beer, 2013; Meyer, Spunt, Berkman, Taylor, \& Lieberman, 2012; Tamir \& Mitchell, 2010; Wagner, Haxby, \& Heatherton, 2012). Moreover, studies have shown that overlapping portions of the MPFC are active when making judgments about similar (Mitchell, Macrae, \& Banaji, 2006), close (Krienen, Tu, \& Buckner, 2010) and familiar others (Visconti di Oleggio Castello, Halchenko, Guntupalli, Gors, \& Gobbini, 2017; Welborn \& Lieberman, 2015). More recently, research on the neural representation of self and person knowledge has turned towards the use of more computational methods, borrowed from the field of machine learning, to better understand how person knowledge might be encoded in patterns of activity as opposed to more traditional measures of relative activation of a brain region (for a review see: Wagner, Chavez, \& Broom, 2018). This work has found evidence that the personality traits that form a part of an individual's representation of other persons can be decoded from activity in the MPFC as individuals retrieve knowledge of those persons (Hassabis et al., 2014; Thornton \& Mitchell, 2018). Although this recent work suggests the MPFC encodes a representation that bears some relationship to a "trait-code", it remains nevertheless unclear if this neural measure of interpersonal perception will follow the same principle as the social psychological findings reviewed above. That is to say, is accuracy in person perception among friends, reflected in the greater correspondence between self/peer trait-judgements, also evident at the level of neural representation? To put it differently, is the information coded within the MPFC during selfreferential thought similarly reflected in the brains of friends when making trait-evaluations about that individual? And if this turns out to be the case, might the degree to which an individual's self/other neural correspondence predict which individuals are more accurately perceived by their friends as evidenced by greater self/other correspondence of personal- 
ity judgements as measured in a separate session?

To our knowledge, these questions have yet to be tested in part because typical neuroimaging studies of self and person knowledge typically employ designs in which there is the same but interpersonally distant target across all participants (e.g. a fictional person, or a celebrity or former president) or the target is interpersonally close to individual participants but necessarily different for each person (e.g. each participant's best friend or family member). Moreover, such studies generally do not simultaneously measure each participant's brain response patterns when evaluating their own self. In the present study, we used a well-established trait-judgment task commonly employed in studies of self and other referential cognition to measure brain response patterns when retrieving information about the self and when thinking about others. Critically we implemented this task in a close-knit social network of peers using a full round-robin design (e.g., Back \& Kenny, 2010) where each participant was both the target stimulus and a perceiver of every other person in the study. In addition, we augmented this design by having an additional condition where participants evaluated their own self-concept in the same way that they evaluate each other. This design allowed us to directly relate the multivoxel response patterns in the MPFC for each individual during selfreferential trait judgments to those obtained during peer trait trait-judgments when that individual was the target. We hypothesized that the similarity between self-referential brain activity and peer brain activity when thinking of that individual would be greater than when peers where thinking about other individuals in their social network. Additionally, we sought to confirm the validity of this self-other neural pattern similarity against behaviorally measured trait ratings collected in a separate session to investigate whether accuracy in person perception, operationalized as greater correspondence between self and peer trait judgments, was reflected in the similarity of neural responses in the MPFC during trait judgments.

\section{Methods}

\section{Participants.}

To accommodate a full round-robin design, we recruited eleven right-handed students ( 5 female) between the ages of 24-29 years old to participate in all aspects of the study. All participants were screened for MRI contraindications, had normal or corrected-to-normal vision, and reported no history of psychiatric or neurological conditions. Participants were recruited from a local student group, representing a close-knit social network in which each person knew every other participant in the study. Each participant in the study participated in two sessions. The first was a behavioral ratings session in which participants filled out personality questionnaires for themselves and their peers. The second session consisted of a scanning session in which participants completed trait judgements for themselves and their peers while undergoing functional neuroimaging. The sample size in this study was targeted as the best compromise between sample size and task length and is similar to previous neuroscientific inves-

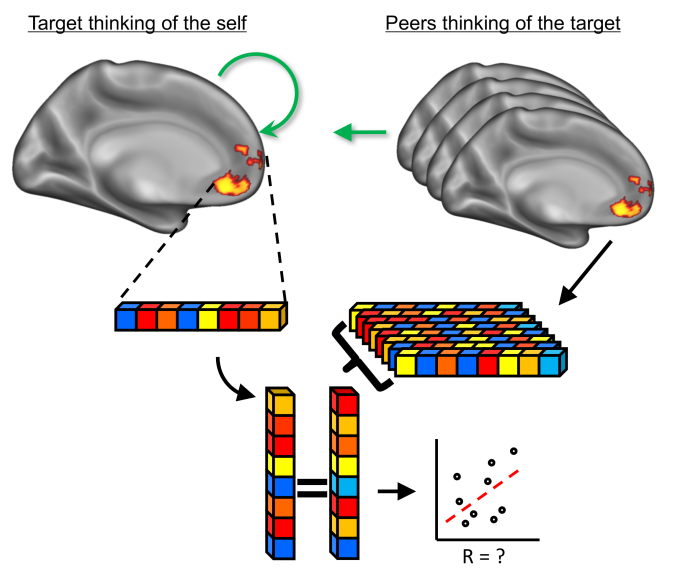

Fig. 1. Schematic of the analysis procedure used in the round-robin self/other task. A region of the MPFC involved in self-referential processing was identified using a functional localizer task. For each participant, voxelwise responses from the MPFC during the round-robin self/other task were extracted for each target participant evaluating their own traits and from all remaining peers when evaluating traits for that same target. These patterns were then aggregated by averaging the voxelwise responses across peers. Finally, for each target, peer responses were correlated with the target's response when thinking of their self.

tigations of real-world dynamic social cognition (Babiloni et al., 2012; Dikker et al., 2017). Owing to the round-robin design used in this study, each additional participant increased the task duration for all participants by approximately four minutes. Thus, a round-robin design as implemented here with 22 participants would have effectively doubled the scan duration (i.e., 150 minutes) for every participant and potentially introduced confounds related to participant fatigue and task habituation. Moreover, the round-robin design used in this study lent itself to a hierarchical linear modelling approach that is better able to model the dependencies in the round-robin design and stands in contrast to the more traditional "summary-statistic" approach that is common in fMRI research and whose statistical power is limited by betweensubject variance. Finally, participants gave informed consent in both sessions in accordance with the guidelines set by the Internal Review Board at The Ohio State University and were compensated for their participation following each session of the study.

\section{Procedures.}

Behavioral Round-Robin Trait Rating Task. Participants were brought into the lab and asked to answer a series of questions about themselves and a set of well-known peers from within their social network. Questions were drawn from common personality inventories in order to cover a number of potential domains of person knowledge, including the Big Five personality traits (Gosling, Rentfrow, \& Swann, 2003), stereotype content model (Fiske, Cuddy, Glick, \& Xu, 2002), and self-esteem (Rosenberg, 1965) scales. Additionally, participants were also asked to give ratings of interpersonal closeness and similarity to each of the targets. All responses were recorded using the PsychoPy stimulus presentation software (Peirce, 2007). During this behavioral trait ratings session, participants were presented with an array of scales (responses 1 to 5), each with the name of one of their peers or 
their own name listed above each. At the top of the screen, a single question was displayed, and participants were asked to give a rating corresponding to impression of their peers or their evaluation of themselves on the given trait. Participants were required to make a rating for every peer on the screen before being allowed to move on to the next question to ensure complete responses. The location of each name was shuffled across trials to avoid order effects with respect to their position on the screen. The entire session lasted approximately an hour. The ratings collected in this session were then used to calculate behavioral self-peer trait rating consensus data for use in subsequent fMRI analyses. In addition to standard frequentist correlation inferences, we also sought to complement this analysis using Bayesian inference by calculating the Bayes factor for comparing the hypothesized linear model against a null model. These test were conducted using the regression function from the BayesFactor package in R (Morey, Rouder, \& Jamil, 2014).

Neuroimaging Round-Robin Trait Rating Task. In a separate session, participants were brought back to the lab to complete the fMRI portion of the experiment. While in the scanner, participants were asked to complete two versions of a standard trait-judgment task widely-used in the study of self-referential processing (Kelley et al., 2002; Mitchell, Schirmer, Ames, \& Gilbert, 2011). The first version of the task was a direct replication of the commonly used trait adjective task (Kelley et al., 2002) in which participants make trait evaluations for themselves, former president Bush or judge whether the word is upper or lower case. This task was used as a functional localizer to independently define the region of MPFC that responds to self to be used in subsequent analysis. In this task, participants were presented with screen containing two words arranged vertically on white text against a black background. For each trial, the top word displayed either "SELF", "BUSH", or "CASE", and the bottom word displayed one of 30 valence-balanced trait adjectives (Anderson, 1968) for $2000 \mathrm{~ms}$ followed by $500 \mathrm{~ms}$ of fixation and intermittent passive fixation trials (2500-7500 ms). Jittered trials were optimized using Opseq2 (Dale, 1999). For the self and Bush trials, participants were asked to make either a "yes" or "no" response using a button box as to whether or not the trait adjective described either themselves or former United States president George W. Bush. During the case condition, participants were simply asked responds "yes" or "no" if the trait adjectives were displayed in all capital letters. One run of this functional localizer task was collected and was used to define a region of interest (ROI) within MPFC that was maximally involved in self-referential processing and which was independent from the main task of interest.

For the main task, we used a similar trait judgment task but modified to accommodate the round-robin nature of the interpersonal perception judgments we collected. We employed a complete round-robin design, meaning that each participant in the study was both a perceiver and target stimulus for every other participant in the study. As with the functional localizer task, for each trial, two words were presented vertically on the screen and participants were asked to make a "yes" or "no" response using the button box. For this version of the task, the top word displayed either "SELF" for trials when making a judgment about the self or the first name of one of the other participants (e.g. "SKYLA", "EVE", "NIKO") in the study. The bottom word was a separate set of 48 valence-balanced trait adjectives (e.g., "HAPPY", "CLUMSY, "SMART"). All targets including the self were presented in every run, and there was a total of eight trails per target per run. Individual traits were only presented once per target randomly across all runs in the experiment. No two participants were presented with the same target/traitadjective order across the experiment, to account for potential order effects.

Neuroimaging Data Acquisition. Magnetic resonance imaging was conducted with a Siemens Magnetom Trio 3.0 Tesla scanner using a 32-channel phased array coil. Structural images were acquired using a T1-weighted MP-RAGE protocol (176 sagittal slices; TR: $1900 \mathrm{~ms}$; TE: $4.4 \mathrm{~ms}$;flip angle:12; $1 \mathrm{~mm}$ isotropic voxels). Functional images were acquired using a T2*-weighted echo planar sequence (TR: $2500 \mathrm{~ms} ; 45$ axial slices, TE: $28 \mathrm{~ms}$; flip angle: $76^{\circ} 3 \mathrm{~mm}$ isotropic voxels. For each participant, we collected one run of the functional localizer task (138 whole-brain volumes) and six runs of the round-robin trait task (160 whole-brain volumes per run). In order to correct for distortion due to B0 inhomogeneity, we also acquired a field map (TR: .512 ms; TE1: .00519, TE2: .00765 , effective echo spacing: .7568). The total length of time for the entire scanning session was approximately 75 minutes.

Self-referential functional localizer task preprocessing and analysis. Functional imaging data for the functional localizer task were preprocessed and voxel responses estimated using FSL (Smith et al., 2004). Data were first slice time corrected followed by a mean-based intensity normalization, high pass filtering (Gaussian-weighted least-squares straight line fitting, with sigma $=100 \mathrm{~s}$ ) and spatially smoothed with a $6 \mathrm{~mm}$ FWHM Gaussian smoothing kernel. Consistent with previous use of this task (Kelley et al., 2002), first-level analyses investigated the self $>$ Bush contrast. These results were then submitted to a group-level random effects analyses to isolate regions of the brain involved in self-referential processing, correcting for multiple comparisons using Thresholdfree Cluster Enhancement (Smith \& Nichols, 2009) in conjunction with 5000 non-parametric permutation tests. Clusters with a corrected $\mathrm{p}$-value $<.05$ from this analysis were then converted into binary masks that were then used to extract self and target response patterns within the MPFC during the round-robin task.

Round-robin self/other task preprocessing and response pattern estimation. Preprocessing and registration of the main self/other task was identical to that of the functional localizer task described above except for the use of a smaller 4mm FWHM Gaussian smoothing kernel (as is more typical 

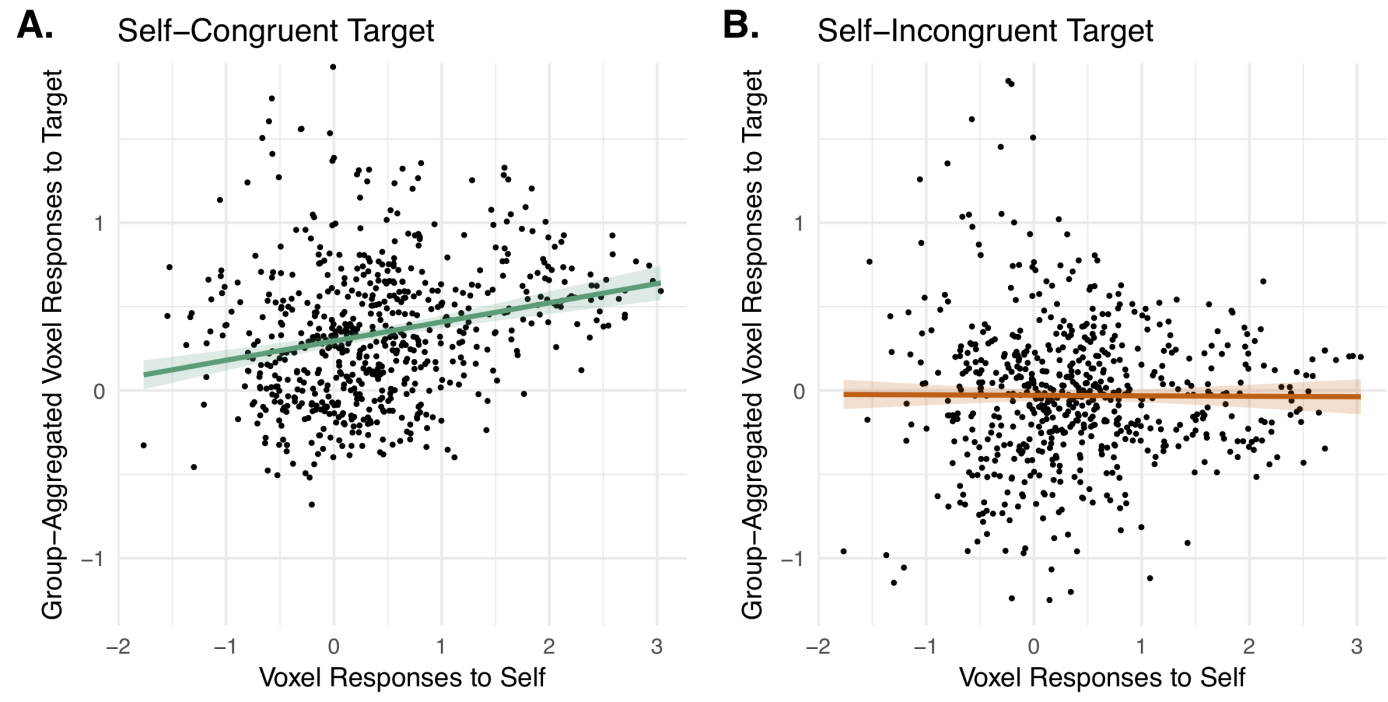

Fig. 2. Illustrative scatter plots from an individual participant depicting the difference between self-congruent targets (A) and self-incongruent targets (B) here represented as simple pattern correlations. Individual points represent voxels within the localized region of interest in the medial prefrontal cortex, with self-referential responses on the $\mathrm{x}$-axis and group-averaged target responses on the $y$-axis. Self-referential brain activity in the target is more strongly associated with the brain activity of friends when they are thinking about the target (i.e. self-congruent) than it is when friends are thinking of a different target (i.e. self-incongruent).

of multivariate fMRI analyses where there is a need to preserve finer-scale neural responses). A multi-step normalization procedure was used to register results to standard space. First, functional data were corrected for spatial distortions using a field map unwarping before aligning functional data to each participant's anatomical scan using boundary based registration (Greve \& Fischl, 2009) in conjunction with a linear registration with FSL's FLIRT tool. These images were then warped to a $2 \mathrm{~mm}$ MNI template using nonlinear registration with FSL's FNIRT tool and a 10mm warp field. All first level task-based analyses were performed in participant's native space before being warped into standard space for final analyses. Parameter estimates were separately estimated for the self condition as well as for each of the ten other peers within each of the six runs. These responses were then combined in a second level within-subject fixed-effects analysis yielding a parameter estimates for the self as well as parameter estimates for of each of the other ten participant's conditions. Normalized (i.e., z-score) voxel responses for each condition within the MPFC ROI defined in the functional localizer task served as input to the subsequent round-robin analysis.

Multivariate round-robin task analysis. For the round-robin multivoxel pattern analysis, voxelwise parameter estimates within each ROI were flattened into a one-dimensional response vectors to allow correlations across participants and conditions. Mirroring classic work in the interpersonal perception literature in which trait ratings are averaged across raters (McCrae \& Costa, 1987), we used aggregated peer response patterns across voxelwise responses yielding a single group averaged response pattern for each target. As is common in studies examining the similarity/dissimilarity of neural representations (e.g., Kriegeskorte, Mur, \& Bandettini, 2008) we computed, for each subject, the dissimilarity between neural response patterns during the self-condition and the group-averaged peer responses vector from the other participants in the study using Spearman rank correlation distance metric. This distance metric is 1-correlation and has the range of $0-2$ with 0 being the least dissimilar (i.e., most similar). The metric is commonly used in various data science and machine learning applications and is used by various unsupervised learning approaches in place of simple correlation A schematic of this analysis approach is displayed in Figure 1 .

Rather than simply testing for a non-zero association between the target's self responses and their peers' responses, we sought to test the more conservative hypothesis which is that these relationships are specific to the given target and, in general, exceed that for other persons (i.e., incongruent targets). To accomplish this, we also computed the correlation between a target's self responses and the group response vector for every other participant in the study. This allowed us to then calculate the similarity between self-congruent target comparisons (i.e. when perceivers were thinking of the same target as self) and self-incongruent target comparisons (i.e. when perceivers were thinking of a different target than the self). Importantly, the incongruent comparisons did not include the self-target responses so as not to influence the dissimilarity with respect to their own congruent patterns. To analyze these data, we fit a multilevel model comparing correlation distances between the self/other responses when congruent versus incongruent with random intercept for each self-target. This model is described here:

$$
\mathrm{Y}_{s t}=\gamma_{00}+u_{t}+\beta_{s t} X_{s t}+e_{s t}
$$

Where $\mathrm{Y}=$ correlation distance, $\gamma=$ fixed intercept, $\mathrm{u}=$ random intercept, $\mathrm{s}=$ subject, $\mathrm{t}=$ target, $\mathrm{X}=$ self-congruent/selfincongruent condition, and $\mathrm{e}=$ the error term. We then compared correlation coefficients for responses between the self and either target-congruent and target-incongruent peer responses via a maximum likelihood multilevel model us- 
ing the lme4 package (Bates, Mächler, Bolker, \& Walker, 2014) with p-values calculated using the lmerTest package (Kuznetsova, Brockhoff, \& Christensen, 2017), both in the $\mathrm{R}$ statistical environment. Self-congruent target versus selfincongruent target effects were compared while allowing random intercepts for within target comparisons. We hypothesized that there would be a greater similarity (i.e. lower correlation distance) for congruent vs. incongruent target comparisons across participants. To compliment the frequentist analyses above, we also tested the same model using a Bayesian multilevel model approach with a Gaussian prior toestimate a $95 \%$-symbo posterior interval around the estimated effect of the model. This was conducted using the Stan language via the 'rstanarm' package in R (Stan Development Team, 2016). Neuroimaging and behavioral data as well as analysis code to replicate the results of this analysis are provided (see open practices section).

Additional brain regions of interest and non-aggregated analyses. Although for our primary analyses we employed an independent localizer task to isolate brain regions within our subjects that were engaged during self-referential processing, it may nevertheless be informative for readers to know to what degree these effects are specific to the vMPFC or whether other areas no identified in the independent localizer task also show evidence of self-other neural similarity.. Thus, we conducted a series of supplementary analyses that replicate the primary round-robin self/other analyses described above in three other ROIs. These ROIs were selected from an automated neuroimaging meta-analysis tool Neurosynth (Yarkoni et al. 2011) are key regions of the social brain that are commonly implicated in self and person knowledge. These are: the posterior cingulate cortex (PCC), the dorsal medial prefrontal cortex (dMPFC), and the right temporal parietal junction (rTPJ). These ROIs were identified from Neurosynth using the term 'self-referential' for the PCC $(z=3.29)$ and the term 'mentalizing' $(z=3.29)$ for the dMPFC and rTPJ.

Additionally, although previous work has demonstrated that aggregating responses across raters leads to better correspondence to self-reported ratings (McCrae \& Costa, 1987), it remains a possibility that aggregating responses across raters may blur useful information about friend pairs that might be recovered in a suitable analysis, albeit with a decreased potential to detect such effects owing to the large decrease in signal-to-noise ratio for individual subject patterns. To address this, we also conducted the primary round-robin analysis in a similar MLM framework without aggregating across peers.

\section{Results}

Round-robin multivoxel self/other similarity in the $v M P F C$. Using a commonly used self/other trait localizer task, we first identified the region of the vMPFC that was active during self-referential thought. Following permutation tests and cluster correction, the results from the group-level analysis of this localizer task identified a single cluster of 641-voxels

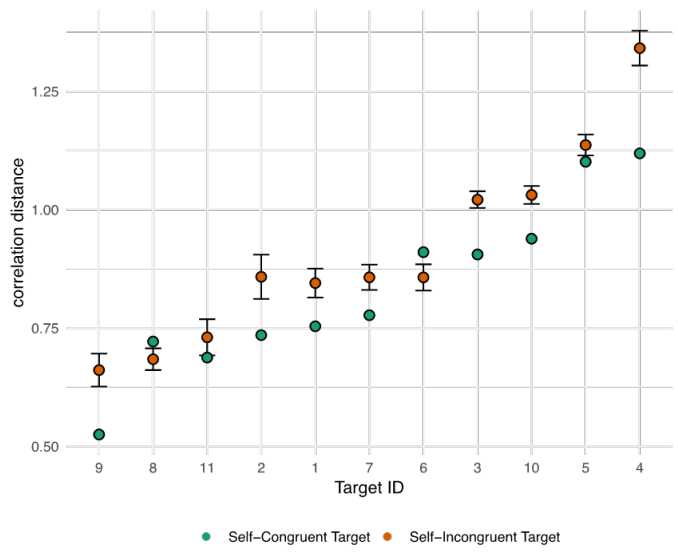

Fig. 3. Within-target dissimilarity (i.e. correlation distance) of perceivers' neural patterns to target's patterns during self-referential thought. Green dots show the dissimilarity between targets thinking about themselves with perceivers thinking about the target (i.e. when the self was congruent with whom the perceivers were thinking). Orange dots show the dissimilarity between targets thinking about themselves with perceivers thinking about different targets (i.e. the self was incongruent with whom the perceivers were thinking), with error bars indicating a one standard error across incongruent targets. As tested in the frequentist and Bayesian linear mixedeffects models, self-congruent patterns showed lower values indicating a greater similarity of self-referential thought to the patterns of perceiver's thoughts when thinking of the congruent target.

centered within the vMPFC $(-8,42,-10)$ which showed significant activation during the self greater than other contrast (see Figure 1). This vMPFC cluster was the only significant cluster from this analysis. From this cluster, a mask of the vMPFC was created and used to extract multivoxel data from the round-robin self/peer trait task. All subsequent analyses were performed within this independently defined region of the vMPFC.

Within the vMPFC ROI defined in the functional localizer task, we calculated the Spearman rank correlation between voxelwise self-referential activation patterns and the group-aggregated voxelwise activation patterns for each target. Rather than simply test whether this correlation is greater than zero, we instead sought to determine if the selfother neural similarity for a given self/target pair (i.e., selfcongruent) was greater than the similarity measure for all self/incorrect target pairs (i.e., self-incongruent) This analysis is a more conservative test of the specificity of self-other neural similarity for a given target person by showing that this similarity measure is greater than what would be expected when a person's self-referential multivoxel response is correlated with the multivoxel response from peers thinking about other persons in their network.

For illustrative purposes, scatter plots from an example participant showing the voxelwise correlations of activity patterns for congruent and incongruent comparisons are displayed in Figure 2A. More generally, across all targets and perceivers in the study, we found that self-congruent target comparisons showed lower dissimilarity (i.e., more similar) $(\mathrm{M}=.834, \mathrm{SD}=.18)$ than self-incongruent target comparisons $(\mathrm{M}=.911, \mathrm{SD}=.22)$, as seen in Figure $2 \mathrm{~B}$. However, in order to properly account for the nested structure of selfcongruent versus self-incongruent comparisons, final inferences were drawn using a linear mixed model while treat- 
ing each target as a random effect. From the linear mixedeffects model, we found that there was a significant difference between the self-congruent target and self-incongruent target comparisons $(\mathrm{b}=-.077, \mathrm{SE}=.03, \mathrm{DF}=110, \mathrm{p}=.011)$. Consistent with our hypothesis, these results show that selfcongruent target dissimilarity was indeed lower (i.e., more similar) than the self-incongruent target dissimilarity when properly accounting for the random intercept for each target (Figure 3). Results of the Bayesian multilevel model showed an effect of $b=-.077,95 \%$ posterior interval [-.137, - .018]. Within the Bayesian framework employed in the current design, this corresponds to a $95 \%$ probability that congruent targets produce neural pattern correlation distance measures that are between $r=.017$ and $r=.137$ smaller (i.e., more similar) than the mean incongruent target distances based on a group of their peers.

Association between neural and behavioral self/other dissimilarity measures. Next, we tested the relationship between the neural self-other dissimilarity measure and a similarly computed self/other agreement score based on the behavioral trait ratings data. Specifically, we tested whether the strength of the neural self-other distance effect was associated with the same distance statistic calculated based on a battery of trait ratings for each participant collected during a separate session. In order to test whether the variability in multivoxel self-other neural distance was associated with self-other distance derived from behavioral ratings, we correlated the fMRI self/other data against those from the behavioral trait ratings and found a significant positive relationship $\mathrm{r}(9)=.71, \mathrm{p}=.014$ such that lower neural distance between self/other (i.e., greater similarity) was associated with lower behavioral trait ratings distance indicated that the more similar self/other neural patterns were with each other, the more accurate peers were at rating the target person's personality. From the Bayesian analyses, we found the relationship between the behavioral and neural accuracy measures to had a Bayes Factor of 4.07 (JZS; Rouder, Speckman, Sun, Morey, \& Iverson, 2009), corresponding to a positive but moderate strength of evidence. Figure 4 displays the relationship between the neural and behavioral data as it relates to each individual participant. These results show that target participants demonstrating greater self/other agreement in neural responses tended to show greater self/other agreement in trait ratings measured independently of the scanning session.

Additional brain regions of interest and non-aggregated analyses results. In addition to the hypothesized role of the multivoxel self-other similarity analyses were also conducted in three additional ROIs to test the specificity of these effects to the functionally localized vMPFC region. Using the identical frequentist linear mixed-effects model, we found no significant differences between self-congruent and self-incongruent comparisons in any of the ROIs: PCC $(\mathrm{b}=-.01, \mathrm{SE}=.02, \mathrm{DF}$ $=110, \mathrm{p}=.57), \mathrm{dMPFC}(\mathrm{b}=.0004, \mathrm{SE}=.02, \mathrm{DF}=110, \mathrm{p}$ $=.97)$, and $\mathrm{rTPJ}(\mathrm{b}=-.048, \mathrm{SE}=.03, \mathrm{DF}=110, \mathrm{p}=.143)$. Bayesian linear mixed effects analyses within these ROIs also

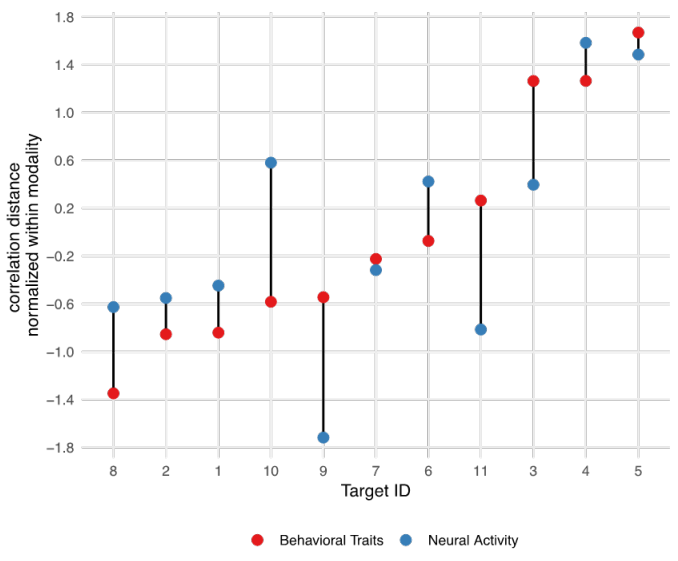

Fig. 4. The relationship between neural and behavioral self-other similarity within each target. Red points depict the normalized behavioral trait self/other correlations and blue points indicate the normalized neural activation correlations during trait evaluations. The black line shows the difference between these measures within each target with shorter lines indicating greater correspondence between behavioral ratings and neural activity. These results show that self/other correspondence in neural activation is correlated with the self/other agreement in trait ratings measured in a separate behavioral session $(r=.71, p=.014$, JZS Bayes Factor $=4.07$ ) such that in general the greater neural self-other correspondence was correlated with greater behavioral self-other correspondence.

showed no $95 \%$ percent posterior intervals that did not include zero: PCC [-0.048, 0.028], dMPFC [-0.047, 0.049], rTPJ $[-0.112,0.017]$. Taken together, both the frequentist and Bayesian analyses showed no evidence of self/other pattern similarity in the PCC, dMPFC, or rTPJ, which are other key regions commonly found in studies of social cognition.

Finally, the primary round-robin self/other analyses were repeated within the vMPFC without aggregating responses across perceivers responses. Although these effects were in the same direction as the aggregated responses, we found no significant differences between self-congruent and selfincongruent comparisons in the frequentist analysis $(\mathrm{b}=$ $.025, \mathrm{SE}=.016, \mathrm{DF}=1089, \mathrm{p}=.139)$ and $95 \%$ posterior interval that included zero [-0.058, 0.008]. Consistent with previous work in personality psychology (McCrae \& Costa, 1987), these results demonstrate the benefits of aggregating responses in benefitting self/other agreement in neural pattern correspondence.

\section{Discussion}

In the present study, we tested whether brain activity patterns during self-referential thought in a target participant were reflected in the brain activity patterns of their peers when evaluating that same target. Using a round-robin design in which each participant was both target and perceiver for every other participant, we show that across participants the multivoxel pattern of brain activity during self-referential processing was more similar to that of peers thinking of this same person than the response associated with peers evaluating other members of their social network. Moreover, we also show that the strength of this neural self-other similarity relationship across the social network is associated with greater accuracy in person perception based on behavioral trait-judgments collected in a separate experimental session. 
Together, these findings provide evidence that the multivoxel pattern of activity in the vMPFC when people reflect on their own self-concept is, to a first approximation, similarly reflected in the vMPFC of well-known peers. Finally, the association between neural self/other similarity and self/other accuracy in trait ratings suggest the possibility that accuracy in person-perception may be predicated on the degree to which an individual's own self-concept is reflected in the multivoxel pattern of activity in the MPFC of their peers.

Functional imaging studies of self and person knowledge have shown consistent evidence that self- and otherreferential thought (e.g., trait evaluations, impression formation, mentalizing) activates the MPFC, with activation in more ventral portions of the MPFC tending to be more associated self-referential processing and more dorsal portions of the MPFC tending to be more associated with person perception and metalizing (Denny, Kober, Wager, \& Ochsner, 2012; Wagner et al., 2012). However, several previous studies have also shown that more ventral aspects of the MPFC are also involved when thinking of other individuals when they are similar to self (Jenkins, Macrae, \& Mitchell, 2008; Mitchell et al., 2006; Tamir \& Mitchell, 2010) or are well-known to the perceiver (Krienen et al., 2010). However, to our knowledge, most studies in this domain relied either on using a target stimulus that is identical across all of the individuals in the study but not known personally (e.g. one or more wellknown celebrities), or alternatively, was well-known to the perceiver but idiosyncratic across all of the participants in the study (e.g., a close friend; (Krienen et al., 2010; Wagner et al., 2019). The round-robin design used in the current study benefits from aspects of both designs by having multiple target persons that are common across participants, however by virtue of the fact that we studied a group of peers, each of targets is also well known to every other person in the social network. The current study also extends previous MVPA findings (Thornton \& Mitchell, 2018; Visconti di Oleggio Castello et al., 2017) by showing that distributed multivoxel activation patterns in the MPFC not only carry identity-specific information but that these neural patterns correspond with those associated with individuals retrieving knowledge about their own self. Moreover, our finding that neural self/other similarity is associated with the accuracy of personality ratings derived from a separate behavioral measure of self and peer trait ratings suggests that not only does the MPFC represent aspects of close-others personality but that the degree to which this representation is similar to that of the target's own self-concept, then these individuals tend to be perceived more accurately within their social network.

In recent years, there has been an increasing number of studies examining physiological and neural synchrony (e.g., Dikker et al., 2017; Parkinson, Kleinbaum, \& Wheatley, 2017; Waters, West, \& Mendes, 2014). This work is conceptually related to the present findings in that these studies have demonstrated that friends and peers show greater neural synchrony when engaged in common tasks (e.g., viewing video clips, engaging in classroom activities). For example, Dikker and colleagues (2017) demonstrated, using portable electroencephalography, that as high-school students perform regular classroom activities, both task engagement and social closeness increases neural synchrony among students. More recently, Parkinson and colleagues found that similarity in neural synchrony, as measured with fMRI, can be used to predict distance between peers in a social network (Parkinson et al., 2017). The present study differs from these conceptually related findings in that we measure the multivariate similarity of spatial neural patterns during retrieval of self and person knowledge. Although both measures can be broadly construed as measures of similarity, it bears noting that they are fundamentally different signals with one having more to do with neuronal populations encoding features of a stimulus and one referring to the synchrony of neuronal timeseries which captures the rise and fall of activity over time in single regions or voxels as a function of a shared task (for a review of these and other methods see Wagner, Chavez \& Broom, 2019).

The interplay between the psychological processes involved in self-referential processing with those involved with processing information about other people has been perennial topic in social perception. Indeed, the study of interpersonal perception seeks to understand the degree to which there is correspondence among different individuals in the beliefs about themselves and one another. For instance, researchers have shown that both self and close others can accurately predict a person's real-world behaviors (Vazire \& Mehl, 2008), that these judgements reflect trait-specific content as well as general affective evaluative factors (Srivastava, Guglielmo, \& Beer, 2010), and is influenced by the number of people within a given group or social network (Kenny \& West, 2010). Until now, there has been very little work linking the interpersonal perception processes described in these behavioral studies to the corresponding patterns of brain activity during these events. Here, we demonstrate that the similarity between self-knowledge and person-knowledge can also be detected at the neural level using fMRI, providing a potential foundation for future studies aimed at examining how the similarity in self/peer neural response may be related to behavioral or mental health outcomes. For example, several studies have attempted to link MPFC activity during selfreferential processing of negative (Yoshimura et al., 2009) or positive (Lemogne et al., 2009) information about the self to identify neural systems implicated in depression and anxiety. However, an alternative way of understanding how an individual's positive or negative view of their own self-concept may be related to mental and social well-being may be to consider the difference between self and peer related brain activity, especially in those cases where the similarity is lowest.

Prior research suggests that length and intimacy of acquaintance are strong moderators of interpersonal accuracy in trait judgments (Connelly \& Ones, 2010; Watson, Hubbard, \& Wiese, 2000). In the present study, participants were recruited from a tight-knit social group and were all familiar with each other. There was, nevertheless, significant variability across individuals in the network in both neural and be- 
havioral self-other agreement. At present, we can only speculate as to why some participants were perceived more accurately than others, prior research argues for the importance of both the perceiver (are they a good judge of character? Are they familiar with the target?) and the target (are they consistent across situations? Are they trying to conceal negative traits?) (Funder, 1995). Another possibility that finds some support in recent work in social neuroscience, is that the participants who are viewed the most accurately are those who are better able to project their personalities to others. Individuals are fundamentally motivated to self-disclose aspects of their personality (Tamir \& Mitchell, 2012), however some individuals may be better able to communicate aspects of themselves than others. For instance, previous studies have shown that when it comes to accurately interpreting the emotions of others, a critical but often overlooked variable is the emotional expressivity of the target (Zaki, Bolger, \& Ochsner, 2008). That is, targets who are more emotionally expressive are viewed more accurately than less expressive targets. Moreover, this empathic accuracy in emotional judgements was also found to be associated with greater activity in approximately the same region of MPFC as shown here in the current study (Zaki, Weber, Bolger, \& Ochsner, 2009). Finally, recent evidence has also suggested that vMPFC activity tracks population consensus of value judgments (Doré et al., 2019), and it is possible that the aggregate vMPFC responses of others thinking of the self are capturing similar collective representations. Future studies will be necessary to tease apart these possibilities.

One limitation of the current study is that the roundrobin nature of the design in conjunction with constraints imposed by the need for robust sampling of neural responses in fMRI analysis limited our ability to study a large sample size. Specifically, each additional participant in a round-robin design increase the task duration for all participants thereby lengthening the duration of an already long session and increasing the possibility of fatigue and task disengagement. That said, the current study can be likened to similar studies where models are tested within a small set of subjects (see: Babiloni et al., 2012; Dikker et al., 2017; Huth, de Heer, Griffiths, Theunissen, \& Gallant, 2016). Moreover, one advantage of the current approach is that it lends itself to a hierarchical linear modeling approach that stands in contrast to the more traditional summary statistic/random effects analysis common in fMRI research and whose power is dominated by between subject variance. Although statistical power is frequently thought of us in terms of sample size (i.e., increasing sample size increases statistical power) in repeated measures designs such as ours, another means of increasing statistical power is to increase the number of measurements. Although this approach might not always be as effective as increasing sample sizes, in situations where constraints of the design limit the sample size (such as ours), increasing the number of measurements can be an efficient way to maximize statistical power (e.g., Bolger, Stadler, \& Laurenceau, 2011). Indeed, in other research using simulated fMRI data with known noise parameters we have shown that, all things be- ing constant, increasing the signal-to-noise ration of parameter estimates can be more effective than increasing sample size when it comes to detecting known effects and rejecting spurious ones (Chavez \& Wagner, 2017). As in many studies, another import limitation to underscore is the use of a single, fixed group of students. This limits the scope of how far these results can be generalized without recruiting more diverse individuals nested within diverse kinds of social relationships (e.g. coworkers, friends, family, adversaries). That said, despite our relatively small sample size, we have designed the study and analysis to ensure our results are as robust as possible given current methods. This includes using an independent functional localizer to avoid whole-brain searches and minimize multiple comparisons, having six fMRI runs of the primary task to improve signal estimation, employing field map corrected image co-registration to decrease measurement error, and the complimentary use of frequentist and Bayesian analyses for inference. Finally, data and analysis code are provided in a public open science repository allowing interested readers to reproduce these analyses and explore the data.

In conclusion, the present study employed multivoxel pattern analysis in conjunction with a novel round-robin experimental design to show that an individual's pattern of brain activity in the VMPFC when retrieving information about the self are reflected in the brains of close peers from their social network when these peers are evaluating that same individual. Moreover, we found that similarity of neural self/other patterns was itself associated with the similarity of self/other trait judgments ratings as measured behaviorally in a separate session. Together, these findings suggest that accuracy in person perception may be predicated on the degree to which a person's self-concept is reflected in the brain activity patterns of well-known individuals within their social network and point to a neural mechanism underlying accuracy interpersonal perception.

\section{ACKNOWLEDGEMENTS}

The authors thank their participants and Allison Londerée for her assistance with this study.

\section{OPEN SCIENCE PRACTICES}

Data, analysis code, and materials have been made publicly available via the Open Science Framework and can be accessed at https://osf.io/vsrxn.

\section{References}

Anderson, N. H. (1968). Likableness ratings of 555 personality-trait words. Journal of Personality and Social Psychology, 9(3), 272-279.

Babiloni, C., Buffo, P., Vecchio, F., Marzano, N., Del Percio, C., Spada, D., ... Perani, D. (2012). Brains "in concert": Frontal oscillatory alpha rhythms and empathy in professional musicians. NeuroImage, 60(1), 105-116. https://doi.org/10.1016/j.neuroimage.2011.12.008

Back, M. D., \& Kenny, D. A. (2010). The Social Relations Model: How to Understand Dyadic Processes. Social and Personality Psychology Compass, 4(10), 855-870. https://doi.org/10.1111/j.1751-9004.2010.00303.x

Bates, D., Mächler, M., Bolker, B., \& Walker, S. 
(2014). Fitting Linear Mixed-Effects Models using lme4. Journal of Statistical Software, 67(1). https://doi.org/10.18637/jss.v067.i01

Bolger, N., Stadler, G., \& Laurenceau, J. (2012). Power analysis for intensive longitudinal measurement designs. In M. R. Mehl \& T. S. Conner (Eds.), Handbook of Research Methods for Studying Daily Life (pp. 285-301).

Chavez, R. S., \& Wagner, D. D. (2017). Mass univariate testing biases the detection of interaction effects in wholebrain analysis of variance. bioRxiv, 130773.

Connelly, B. S., \& Ones, D. S. (2010). An other perspective on personality: Meta-analytic integration of observers' accuracy and predictive validity. Psychological Bulletin, 136(6), 1092-1122. https://doi.org/10.1037/a0021212

Dale, A. M. (1999). Optimal experimental design for event-related fMRI. Human Brain Mapping, 8(2-3), 109-114. https://doi.org/10.1002/(SICI)10970193(1999)8:2/3<109::AID-HBM7>3.0.CO;2-W

Denny, B. T., Kober, H., Wager, T. D., \& Ochsner, K. N. (2012). A meta-analysis of functional neuroimaging studies of self- and other judgments reveals a spatial gradient for mentalizing in medial prefrontal cortex. Journal of Cognitive Neuroscience, 24(8), 1742-1752. https://doi.org/10.1162/jocn ${ }_{a 0} 0233$

Dikker, S., Wan, L., Davidesco, I., Kaggen, L., Oostrik, M., McClintock, J., ... Poeppel, D. (2017). Brainto-Brain Synchrony Tracks Real-World Dynamic Group Interactions in the Classroom. Current Biology, 27(9), 1375-1380. https://doi.org/10.1016/j.cub.2017.04.002

Doré, B. P., Scholz, C., Baek, E. C., Garcia, J. O., O'Donnell, M. B., Bassett, D. S., ... Falk, E. B. (2019). Brain Activity Tracks Population Information Sharing by Capturing Consensus Judgments of Value. Cerebral Cortex, 29(7), 3102-3110. https://doi.org/10.1093/cercor/bhy176

Fiske, S. T., Cuddy, A. J. C., Glick, P., \& Xu, J. (2002). A model of (often mixed) stereotype content: Competence and warmth respectively follow from perceived status and competition. Journal of Personality and Social Psychology, 82(6), 878-902. https://doi.org/10.1037/00223514.82.6.878

Funder, D. C. (1995). On the accuracy of personality judgment: A realistic approach. Psychological Review, 102(4), 652-670. https://doi.org/10.1037/0033295X.102.4.652

Funder, D. C., \& Colvin, C. R. (1988). Friends and Strangers: Acquaintanceship, Agreement, and the Accuracy of Personality Judgment. Journal of Personality and Social Psychology, 55(1), 149-158. https://doi.org/10.1037/0022-3514.55.1.149

Gobbini, M. I., Gors, J. D., Halchenko, Y. O., Hughes, H. C., \& Cipolli, C. (2013). Processing of invisible social cues. Consciousness and Cognition, 22(3), 765-770. https://doi.org/10.1016/j.concog.2013.05.002

Gosling, S. D., Rentfrow, P. J., \& Swann, W. B. (2003). A very brief measure of the Big-Five personality domains.
Journal of Research in Personality, 37(6), 504-528. https://doi.org/10.1016/S0092-6566(03)00046-1

Greve, D. N., \& Fischl, B. (2009). Accurate and robust brain image alignment using boundarybased registration. NeuroImage, 48(1), 63-72. https://doi.org/10.1016/j.neuroimage.2009.06.060

Hassabis, D., Spreng, R. N., Rusu, A. a, Robbins, C. a, Mar, R. a, \& Schacter, D. L. (2014). Imagine All the People: How the Brain Creates and Uses Personality Models to Predict Behavior. Cerebral Cortex, 24(8), 1979-1987. https://doi.org/10.1093/cercor/bht042

Hughes, B. L., \& Beer, J. S. (2013). Protecting the self: the effect of social-evaluative threat on neural representations of self. Journal of Cognitive Neuroscience, 25(4), 613-622.

Huth, A. G., de Heer, W. A., Griffiths, T. L., Theunissen, F. E., \& Gallant, J. L. (2016). Natural speech reveals the semantic maps that tile human cerebral cortex. Nature, 532(7600), 453-458. https://doi.org/10.1038/nature17637

Jenkins, A. C., Macrae, C. N., \& Mitchell, J. P. (2008). Repetition suppression of ventromedial prefrontal activity during judgments of self and others. Proceedings of the National Academy of Sciences, 105(11), 4507-4512. https://doi.org/10.1073/pnas.0708785105

John, O. P., \& Robins, R. W. (1993). Determinants of Interjudge Agreement on Personality Traits: The Big Five Domains, Observability, Evaluativeness, and the Unique Perspective of the Self. Journal of Personality, 61(4), 521-551. https://doi.org/10.1111/j.14676494.1993.tb00781.x

Kelley, W. M., Macrae, C. N., Wyland, C. L., Caglar, S., Inati, S., \& Heatherton, T. F. (2002). Finding the self? An event-related fMRI study. Journal of Cognitive Neuroscience, 14(5), 785-794.

Kenny, D. A., \& West, T. V. (2010). Similarity and Agreement in Self-and Other Perception: A Meta-Analysis. Personality and Social Psychology Review, 14(2), 196-213. https://doi.org/10.1177/1088868309353414

Kolar, D. W., Funder, D. C., \& Colvin, C. R. (1996). Comparing the accuracy of personality judgements by the self and knowledgeable others. Journal of Personality, 64(2), 311-337. Retrieved from http://www.ncbi.nlm.nih.gov/pubmed/8656321

Kriegeskorte, N., Mur, M., \& Bandettini, P. (2008). Representational similarity analysis - connecting the branches of systems neuroscience. Frontiers in Systems Neuroscience, 2, 4. https://doi.org/10.3389/neuro.06.004.2008

Krienen, F. M., Tu, P.-C., \& Buckner, R. L. (2010). Clan mentality: evidence that the medial prefrontal cortex responds to close others. The Journal of Neuroscience, 30(41), 13906-13915. https://doi.org/10.1523/JNEUROSCI.2180-10.2010

Kuznetsova, A., Brockhoff, P. B., \& Christensen, R. H. B. (2017). ImerTest Package: Tests in Linear Mixed Effects Models. Journal of Statistical Software, 82(13). 
https://doi.org/10.18637/jss.v082.i13

Lemogne, C., le Bastard, G., Mayberg, H., Volle, E., Bergouignan, L., Lehé;ricy, S., ... Fossati, P. (2009). In search of the depressive self: Extended medial prefrontal network during self-referential processing in major depression. Social Cognitive and Affective Neuroscience. https://doi.org/10.1093/scan/nsp008

McCrae, R. R., \& Costa, P. T. (1987). Validation of the Five-Factor Model of Personality Across Instruments and Observers. Journal of Personality and Social Psychology, 52(1), 81-90. https://doi.org/10.1037/00223514.52.1.81

Meyer, M. L., Spunt, R. P., Berkman, E. T., Taylor, S. E., \& Lieberman, M. D. (2012). Evidence for social working memory from a parametric functional MRI study. Proceedings of the National Academy of Sciences, 109(6), 1883-1888. https://doi.org/10.1073/pnas.1121077109

Mitchell, J. P., Macrae, C. N., \& Banaji, M. R. (2006). Dissociable medial prefrontal contributions to judgments of similar and dissimilar others. Neuron, 50(4), 655-663. https://doi.org/10.1016/j.neuron.2006.03.040

Mitchell, J. P., Schirmer, J., Ames, D. L., \& Gilbert, D. T. (2011). Medial prefrontal cortex predicts intertemporal choice. Journal of Cognitive Neuroscience, 23(4), 857-866. https://doi.org/10.1162/jocn.2010.21479

Morey, R. D., Rouder, J. N., \& Jamil, T. (2014). BayesFactor: Computation of Bayes factors for common designs. $\mathrm{R}$ Package Version 0.9.

Parkinson, C., Kleinbaum, A. M., \& Wheatley, T. (2017). Spontaneous neural encoding of social network position. Nature Human Behaviour, 1(5), 0072. https://doi.org/10.1038/s41562-017-0072

Peirce, J. W. (2007). PsychoPy-Psychophysics software in Python. Journal of Neuroscience Methods, 162(1-2), 8-13. https://doi.org/10.1016/j.jneumeth.2006.11.017

Rosenberg, M. (1965). Society and the adolescent selfimage. Princeton, NJ: Princeton University.

Rouder, J. N., Speckman, P. L., Sun, D., Morey, R. D., \& Iverson, G. (2009). Bayesian t tests for accepting and rejecting the null hypothesis. Psychonomic Bulletin \& Review, 16(2), 225-237. https://doi.org/10.3758/PBR.16.2.225

Smith, S. M., Jenkinson, M., Woolrich, M. W., Beckmann, C. F., Behrens, T. E. J., Johansen-Berg, H., ... Matthews, P. M. (2004). Advances in functional and structural MR image analysis and implementation as FSL. NeuroImage, 23, S208-S219. https://doi.org/10.1016/j.neuroimage.2004.07.051

Smith, S. M., \& Nichols, T. E. (2009). Thresholdfree cluster enhancement: addressing problems of smoothing, threshold dependence and localisation in cluster inference. NeuroImage, 44(1), 83-98. https://doi.org/10.1016/j.neuroimage.2008.03.061

Srivastava, S., Guglielmo, S., \& Beer, J. S. (2010). Perceiving others' personalities: examining the dimensionality, assumed similarity to the self, and stability of perceiver effects. Journal of Personality and Social Psychology,
98(3), 520-534. https://doi.org/10.1037/a0017057

Stan Development Team. (2016). rstanarm: Bayesian applied regression modeling via Stan. $\mathrm{R}$ package version 2.13.1. R Package Version. Retrieved from https://cran.r-project.org/package=rstanarm

Tamir, D. I., \& Mitchell, J. P. (2010). Neural correlates of anchoring-and-adjustment during mentalizing. Proceedings of the National Academy of Sciences of the United States of America, 107(24), 10827-10832. https://doi.org/10.1073/pnas.1003242107

Tamir, D. I., \& Mitchell, J. P. (2012). Disclosing information about the self is intrinsically rewarding. Proceedings of the National Academy of Sciences, 109(21), 8038-8043. https://doi.org/10.1073/pnas.1202129109

Thornton, M. A., \& Mitchell, J. P. (2018). Theories of Person Perception Predict Patterns of Neural Activity During Mentalizing. Cerebral Cortex, 28(10), 3505-3520. https://doi.org/10.1093/cercor/bhx216

Vazire, S., \& Mehl, M. R. (2008). Knowing me, knowing you: the accuracy and unique predictive validity of selfratings and other-ratings of daily behavior. Journal of Personality and Social Psychology, 95(5), 1202-1216. https://doi.org/10.1037/a0013314

Visconti di Oleggio Castello, M., Halchenko, Y. O., Guntupalli, J. S., Gors, J. D., \& Gobbini, M. I. (2017). The neural representation of personally familiar and unfamiliar faces in the distributed system for face perception. Scientific Reports, 7(1), 12237. https://doi.org/10.1038/s41598-017-12559-1

Wagner, D. D., Chavez, R. S., \& Broom, T. W. (2019). Decoding the neural representation of self and person knowledge with multivariate pattern analysis and data-driven approaches. Wiley Interdisciplinary Reviews: Cognitive Science, 10(1), e1482. https://doi.org/10.1002/wcs.1482

Wagner, D. D., Haxby, J. V, \& Heatherton, T. F. (2012). The Representation of Self and Person Knowledge in the Medial Prefrontal Cortex. Wiley Interdisciplinary Reviews: Cognitive Science, 3(4), 451-470. https://doi.org/10.1002/wcs.1183

Waters, S. F., West, T. V., \& Mendes, W. B. (2014). Stress Contagion. Psychological Science, 25(4), 934-942. https://doi.org/10.1177/0956797613518352

Watson, D., Hubbard, B., \& Wiese, D. (2000). Self-other agreement in personality and affectivity: The role of acquaintanceship, trait visibility, and assumed similarity. Journal of Personality and Social Psychology, 78(3), 546-558. https://doi.org/10.1037/0022-3514.78.3.546

Welborn, B. L., \& Lieberman, M. D. (2015). Personspecific Theory of Mind in Medial pFC. Journal of Cognitive Neuroscience, 27(1), 1-12. https://doi.org/10.1162/jocn ${ }_{a 0} 0700$

Yoshimura, S., Ueda, K., Suzuki, S. ichi, Onoda, K., Okamoto, Y., \& Yamawaki, S. (2009). Selfreferential processing of negative stimuli within the ventral anterior cingulate gyrus and right amygdala. Brain and Cognition, 69(1), 218-225. 
https://doi.org/10.1016/j.bandc.2008.07.010

Zaki, J., Weber, J., Bolger, N., \& Ochsner, K. (2009). The neural bases of empathic accuracy. Proceedings of the National Academy of Sciences, 106(27), 11382-11387. https://doi.org/10.1073/pnas.0902666106

Zaki, Jamil, Bolger, N., \& Ochsner, K. (2008). It takes two: The interpersonal nature of empathic accuracy: Research article. Psychological Science, 19(4), 399-404. https://doi.org/10.1111/j.1467-9280.2008.02099.x 